\title{
Anemia hemolítica inmunomediada (AHIM)
}

\section{Immune-mediated hemolytic anemia (IMHA)}

\author{
Ruiz Chipo Carol Katherine ${ }^{1}$; Benavides Insignares Henry ${ }^{2}$ y \\ Roque Rodríguez Anita Isabel ${ }^{3}$ \\ ${ }^{1}$ MVZ Unillanos, ${ }^{2}$ MV ULS y ${ }^{3}$ MV MSc Docente Unillanos \\ anita.roque@unillanos.edu.co
}

Recibido 04 de Febrero 2014, Aceptado 25 de Abril 2014

\section{RESUMEN}

La anemia hemolítica inmunomediada (AHIM) canina resulta de la destrucción prematura de eritrocitos debido a la fijación de anticuerpos a antígenos propios o extraños ubicados sobre la membrana celular; la anemia aparece por la incapacidad de la médula ósea para compensar la elevada destrucción eritrocitaria. Esta enfermedad afecta con mayor frecuencia a hembras de edad media y de todas las razas. Dentro de las manifestaciones clínicas suele encontrarse anorexia, mucosas pálidas o ictéricas, soplo cardiaco, hepatoesplenomegalia, taquipnea, taquicardia y hematuria entre otras. Los hallazgos en el examen físico y pruebas paraclínicas son el reflejo de la hipoxia tisular causada por la anemia; además de alteraciones hemostáticas si hay trombocitopenia concurrente. La AHIM se confirma de dos maneras: 1) la presencia de anemia, autoglutinación y esferocitosis en el extendido hemático, y 2) la prueba de Coombs directa; la cual detecta los anticuerpos sobre las membranas eritrocitarias, confirmando el diagnóstico. Las principales complicaciones son la coagulación intravascular diseminada (CID) y la enfermedad tromboembólica, en particular el tromboembolismo pulmonar (TEP). El tratamiento de la AHIM canina se basa en la administración de corticosteroides con o sin alquilantes, junto a la terapia de sostén necesaria en cada uno de los casos, además se debe controlar o eliminar la enfermedad primaria. En general la mortalidad de los pacientes 
caninos con AHIM varía del 20 al 50\%, pero puede ser más elevada en los cuadros fulminantes y complicados.

Palabras claves: Anemia hemolítica, caninos, sangre.

\begin{abstract}
The immune-mediated hemolytic canine anemia (IMHA) is caused by the early destruction of erythrocytes due to the fixation of antibodies to own or foreign antigens on cell membrane surface; consequently, the anemia appears to be a result of the inability of the bone marrow to compensate the high red cell destruction. This disease affects females of medium age with major frequency and to every breed. The clinic manifestations usually are on physical examination and complementary test showing the hypoxia tissue caused by the anemia; as well as haemostatic alterations if there is concurrent thrombocytopenia. The IMHA can be confirmed by two ways: 1) the presence of anemia, autoagglutination and spherocytosis in the blood smear, and 2) the direct test of Coombs. The latter detects the antibodies on the erythrocyte membranes. The main complications are the disseminated intravascular coagulation (DIC) and thromboembolic disease, in particular the pulmonary thromboembolism (PTE). The treatment of the canine IMHA is based on the administration of corticosteroid with or without alkylating, together with the therapy of support necessary in each one of the cases, and controlling or eliminating the primary disease. In general, the death rate of the canine patients with IMHA change from 20 to $50 \%$, but it can be higher than in fulminating and complicated presentations.
\end{abstract}

Keywords: Hemolytic anemia, canines, blood.

\title{
INTRODUCCIÓN
}

La anemia se define como la disminución en el número de glóbulos rojos en circulación, la cual se origina debido a: 1) la disminución de la producción de eritrocitos a nivel de la medula ósea, 2) pérdida de sangre entera por hemorragia, o 3) por acortamiento de la vida media del eritrocito por hemólisis, la cual puede 
estar mediada o no por el sistema inmune. La anemia hemolítica inmunomediada (AHIM), hace referencia a la destrucción de eritrocitos debido a la fijación de anticuerpos (IgG y/o IgM) a antígenos propios (AHIM idiopática o autoinmune) o extraños (AHIM secundaria) que se encuentran sobre la superficie de la membrana celular, permitiendo la subsiguiente fijación del complemento y la lisis intravascular (reacción citotóxica o de hipersensibilidad tipo II), o cubriendo (opsonización) los eritrocitos (Figura 1) lo que permite su destrucción por parte de los macrófagos en el bazo y el hígado principalmente (hemólisis extravascular). La anemia aparece por la incapacidad del organismo para compensar la elevada destrucción eritrocitaria, dando lugar a manifestaciones clínicas acordes con la hipoxia tisular (Thompson, 1996).

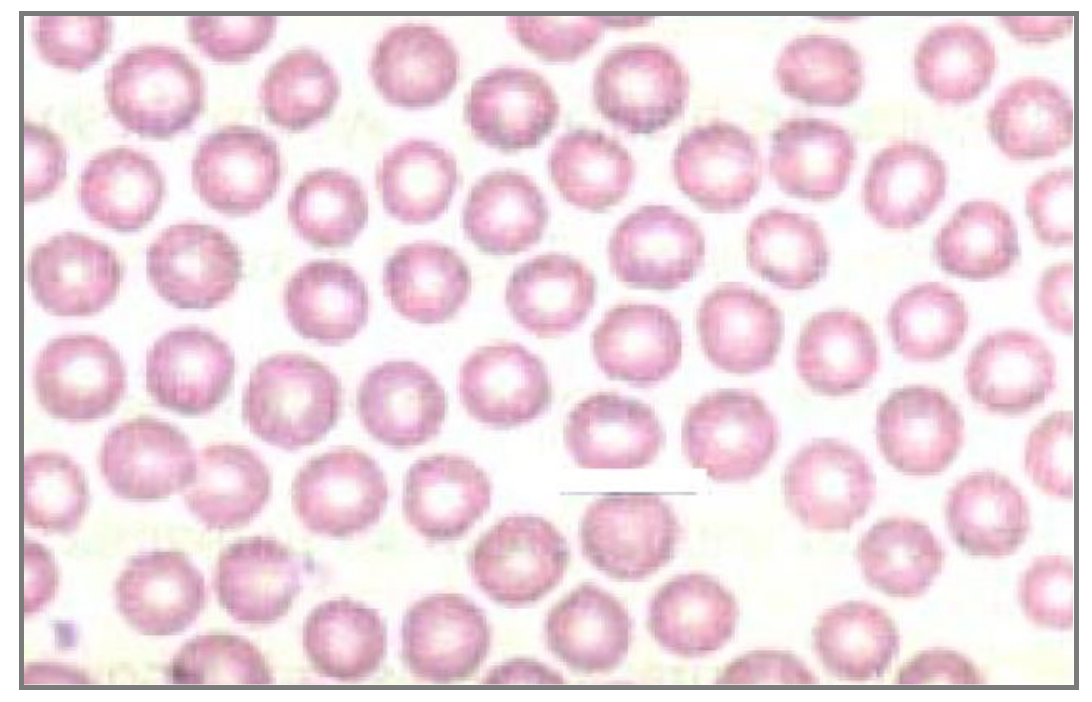

Figura 1. Eritrocito canino. Tinción Wrigth 100x. Fuente: Moreno, 2003

La Anemia hemolítica inmunomediada (AHIM) (Figura 2) es el proceso inmunológico más prevalente en los caninos y constituye aproximadamente el $20 \%$ del total de las anemias reportadas en esta especie (Olarte, 2004). La AHIM es el resultado de la destrucción prematura de los glóbulos rojos debido a la fijación de anticuerpos ( $\lg G$, $\lg M$ y/o complemento) a antígenos propios o extraños ubicados sobre la superficie de su membrana celular, apareciendo la anemia por la incapacidad de la médula ósea para compensar la elevada destrucción. 


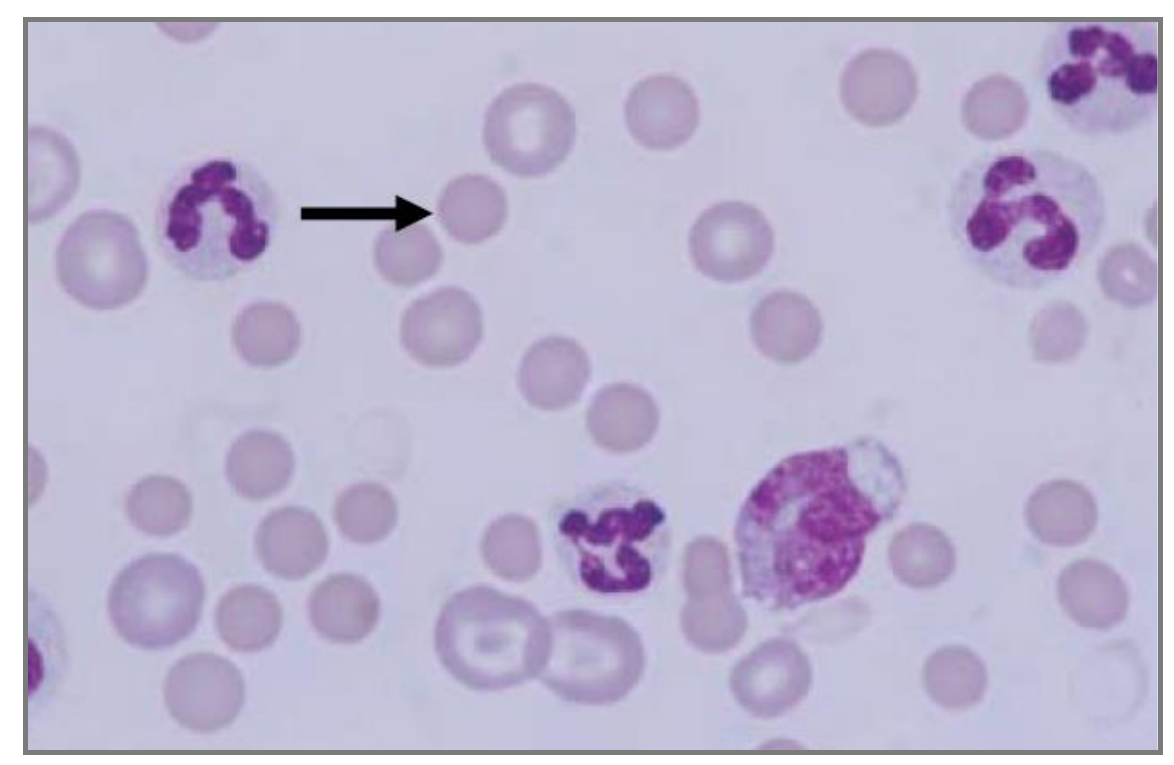

Figura 2. Anemia hemolítica inmunomediada canina, numerosos esferocitos.

Tinción Wrigth 100x. Fuente: Rebar, 2003

\section{CLASIFICACIÓN Y ETIOLOGÍA DE LA AHIM CANINA}

La AHIM se puede clasificar de tres maneras: 1) según el lugar donde ocurre la hemólisis como intravascular (enfermedad grave y de carácter agudo) o extravascular (ocurre en el bazo y el hígado principalmente, es de presentación crónica); 2) según su etiología puede ser primaria o idiopática cuando no se puede determinar la causa detallada del padecimiento $(65-75 \%$ de los casos) 0 secundaria cuando se presenta por consecuencia de otra enfermedad subyacente; y 3) según el tipo de inmunoglobulina participante, la temperatura y la naturaleza del proceso hemolítico, se puede clasificar en cinco clases que se resumen en la Tabla 1 (Tizard, 2002).

La AHIM primaria denota la perdida de la autotolerancia o incapacidad del sistema inmune de diferenciar los antígenos propios de los extraños y equivale a un disturbio primario de dicho sistema (Lifton, 2000). También, la AHIM secundaria puede originarse como consecuencia de otras enfermedades: lupus eritematoso sistémico (LES); neoplasias como el linfosarcoma, hemangiosarcoma y leucemia linfoide; otra manera de adquirir la AHIM es por medicación con cefalosporinas, 
penicilinas, tetraciclinas, sulfonamidas y vacunación. Además de la reacción inmune a parásitos como Babesia, Mycoplasma, Dirofilaria, Ehrlichia y Leishmania, y post transfusión de sangre entera o células (Paz, 2004).

Tabla 1. Clasificación de la AHIM canina según el tipo de anticuerpo participante, la temperatura óptima a la que reaccionan estos anticuerpos (Ac) y la naturaleza del proceso hemolítico.

\begin{tabular}{|c|c|c|c|c|c|}
\hline Clase & $\begin{array}{c}\text { Anticuerpo } \\
\text { predominante }\end{array}$ & Actividad & $\begin{array}{c}\text { Temperatura } \\
\text { optima }\left({ }^{\circ} \mathrm{C}\right)\end{array}$ & $\begin{array}{c}\text { Sitio de } \\
\text { eliminación } \\
\text { eritrocitos }\end{array}$ & $\begin{array}{l}\text { Efecto } \\
\text { clínico }\end{array}$ \\
\hline I & $\mathrm{G}>\mathrm{M}$ & Aglutinina & 37 & Bazo & $\begin{array}{l}\text { Aglutinación } \\
\text { intravascular }\end{array}$ \\
\hline II & $\mathrm{M}$ & Hemolisina & 37 & Hígado & $\begin{array}{l}\text { Hemólisis } \\
\text { intravascular }\end{array}$ \\
\hline III & G & $\begin{array}{c}\text { Ac. } \\
\text { incompleto }\end{array}$ & 37 & Bazo & Anemia \\
\hline IV & $M$ & Aglutinina & 4 & Hígado & $\begin{array}{l}\text { Cianosis de } \\
\text { extremidades }\end{array}$ \\
\hline V & M & $\begin{array}{c}\text { Ac. } \\
\text { incompleto }\end{array}$ & 4 & Hígado & Anemia \\
\hline
\end{tabular}

Fuente: Tizard, (2002).

\section{INMUNOPATOGENIA DE LA ANEMIA HEMOLÍTICA INMUNOMEDIADA CANINA (AHIM)}

Esta enfermedad se caracteriza por la elevada destrucción de los glóbulos rojos causada por la fijación de las inmunoglobulinas (IgG y/o $\operatorname{lgM}$ ) y el complemento a la membrana del eritrocito (mecanismo de hipersensibilidad tipo II - citotoxicidad mediada por anticuerpos) (Reimer et al., 2000). Se debe tener en cuenta que la IgM causa menos del $1 \%$ de los casos de AHIM (Nelson y Couto, 2000) y, que cuando el complemento se encuentra sólo sobre la membrana celular se produce una enfermedad diferente a la AHIM donde la hemólisis marcada es rara (Lifton, 2000; Paz, 2004).

Se han postulado varios mecanismos mediante los cuales el anticuerpo se puede unir al eritrocito dando origen a la AHIM, estos mecanismos son: 
1) Autoinmune: cuando en la enfermedad primaria un anticuerpo se une a un determinante estructural de membrana. El auto-anticuerpo puede desarrollarse tras una infección por un microbio portador de un epitopo de reacción cruzada, o que induce hiperactividad del sistema inmune o activación de linfocitos autorreactivos (Lifton, 2000).

2) Aloinmune: aparece de manera espontánea cuando un alo-anticuerpo se une al antígeno de grupo sanguíneo, que generalmente son inducidos por una transfusión de sangre incompatible, o ser traspasados a los recién nacidos a través del calostro (Isoeritrólisis neonatal) (Day, 2000).

3) Espectador inocente: cuando un anticuerpo se une a un epitopo extraño portado por un agente infeccioso o un fármaco por ejemplo la penicilina que va unida de forma inespecífica a la superficie del eritrocito (Lifton, 2000; Paz, 2004).

4) Automodificado: sucede cuando un fármaco actúa como un hapteno que se une a una molécula de la superficie del eritrocito creando un nuevo epitopo (Day, 2000).

5) Determinante críptico expuesto: se presenta con la unión de un fármaco o un microorganismo que provoca la exposición de un determinante que hasta el momento había sido críptico. En este caso el anticuerpo es un autoanticuerpo, pero la enfermedad es secundaria a la causa desencadenante, adicionalmente, los complejos inmunes (Igs) pueden adsorber de forma inespecífica sobre la superficie del eritrocito que no es mediante un enlace Fab, aunque no necesariamente, median la destrucción celular (Lifton, 2000; Day, 1999).

\section{DESTRUCCIÓN CELULAR EN LA AHIM}

La iniciación de la AHIM implica la presentación de un antígeno propio o extraño (reacción cruzada) por las moléculas complejo mayor de histocompatibilidad $(\mathrm{MHC})$ e interacciones entre linfocitos autorreactivos $\mathrm{T}$ y $\mathrm{B}$, lo que da lugar a un 
auto-anticuerpo. Estos eritrocitos cubiertos con anticuerpos se unen a receptores Fc sobre los macrófagos y monocitos del sistema fagocítico mononuclear (SFM), con la resultante fagocitosis (Semple y Freedman, 2005), esta hemólisis extravascular ocurre principalmente en el bazo (células cubiertas con lgG) y en menor proporción en el hígado (células cubiertas con $\operatorname{lgM}$ y complemento) (Nelsom y Couto, 2000; Cerecedo et al., 2004), si el macrófago elimina solo una parte de la membrana celular, el eritrocito pierde su configuración bicóncava usual y forma una célula densa pequeña denominada esferocito (Buch, 1999). Estos esferocitos carecen de flexibilidad y son más susceptibles de ser eliminados por los macrófagos esplénicos o hepáticos, o de destruirse al pasar por los pequeños capilares (Cowell et al., 2003; Herrera y Estrada, 2003).

Por otro lado, y con menor frecuencia, los eritrocitos cubiertos con anticuerpos se eliminan mediante hemólisis intravascular tras la fijación del complemento (Day, 2000). También en ocasiones los auto-anticuerpos se dirigen contra los precursores eritroideos de la médula ósea, destruyéndolos en el estadio de metarrubricito o reticulocito (arresto en la maduración eritroide), en este caso se produce una forma grave de AHIM no regenerativa produciendo aplasia de glóbulos rojos pura (PRCA) (Nassiri et al., 2005). Los perros con PRCA pueden no tengan anticuerpos unidos con los eritrocitos circulantes, pero la lgG sérica puede inhibir la eritropoyesis uniéndose a dichos precursores o a la eritropoyetina en la médula ósea, siendo este padecimiento es un tipo de AHIM, del cual existen reportes que indican que la mayoría de los pacientes con PRCA necesitan tratamiento de por vida (Álvarez, 2006).

La formación de células B autorreactivas contribuye a la cronicidad de la respuesta autoinmune, esto incrementa el número de estas células. En el tejido inflamado, citoquinas proinflamatorias y auto-antígenos proveen las señales que favorecen la diferenciación y sobrevivencia de las células plasmáticas, los anticuerpos producidos localmente por estas células contribuyen a la severidad de la inflamación, además, las células plasmáticas proporcionan persistencia de la memoria humoral, estas son de larga vida y son relativamente resistentes a las 
terapias existentes de inmunosupresores y continúan secretando anticuerpos sin necesidad de estimulación. Estas células proporcionan títulos de anticuerpos constantes durante la fase clínica y luego de la terapia inmunosupresora, estos títulos persisten, aunque a menudo bajos y no causan signos clínicos agudos, es probable que mantenga un bajo nivel de inflamación crónica y destrucción de tejidos progresiva, con disminución del umbral de ruptura de la tolerancia (Manz et al., 2006).

\section{Características inmunopatológicas de la anemia hemolítica autoinmune}

1. En la superficie del eritrocito se pueden identificar auto-anticuerpos para las clases de IgG con cuatro subclases, aunque pueden predominar la lgG1 e IgG4, también se puede presentar lgM (Wilkerson et al., 2000).

2. La mayor parte del auto-anticuerpo está unido al eritrocito siendo la proporción circulante libre muy baja.

3. Se ha demostrado la especificidad de los autoanticuerpos en la AHIM canina tomando anticuerpos de eritrocitos de pacientes y usándolos para inmunoprecipitar autoantígenos de glóbulos rojos caninos, las principales moléculas autoantigénicas incluyen las glicoforinas y los componentes del canal de intercambio aniónico del eritrocito (banda 3) (Barker et al., 2002). En los pacientes con AHIM se identifican autoanticuerpos específicos para la molécula citoesquelética espectrina, así como en los perros normales, por lo que podrían participar en el proceso de eliminación de los glóbulos rojos viejos. La heterogeneidad de la respuesta de los anticuerpos sugiere que la etiología subyacente a la AHIM puede variar de un individuo a otro (Day, 2000).

4. Al comparar la fagocitosis de eritrocitos opsonizados in vitro es mayor con monocitos derivados de perros que presentan AHIM que con monocitos de perros normales (Corato et al., 1997). 
5. En ocasiones, se encuentran células autorreactivas $T$ (especificas para proteínas de membrana de glóbulos rojos y péptidos de glucoforina) en la sangre de perros clínicamente normales, pero se identifican con frecuencia en la sangre de pacientes con AHIM, o en animales que, aunque están clínicamente sanos tienen susceptibilidad genética a esta enfermedad (Day, 2000; Corato et al., 1997).

6. La magnitud de la hemólisis depende del título de anticuerpos; la afinidad del anticuerpo por el autoantígeno y su capacidad fijar el complemento, la densidad antigénica sobre la membrana y el estado de activación de linfocitos (Lifton, 2000).

\section{ASPECTOS CLÍNICOS DEL PACIENTE CANINO CON AHIM}

Reseña. La AHIM afecta con mayor regularidad a las hembras con edades entre los 2 a 8 años y afecta a cualquier raza.

Manifestaciones clínicas y anamnesis. Los caninos con AHIM manifiestan depresión, anorexia, debilidad, letargia, vómito y diarrea. El colapso o sincope es un signo menos común, y unos pocos perros pueden presentar disnea antes de cualquier otro signo (Day y Mackin, 2000).

Examen físico. Los hallazgos incluyen mucosas pálidas o ictéricas, fiebre, hepatoesplenomegalia, taquipnea, taquicardia y soplo cardiaco (Paz, 2004). Otras anormalidades incluyen bilirrubinuria o hemoglobinuria. Además, petequias, equimosis, melena y epistaxis si hay trombocitopenia (Olarte, 2004)

\section{ESTUDIOS DIAGNÓSTICOS}

Inicialmente se debe determinar el tipo de anemia, si es por disminución de la eritropoyesis, hemorragia o hemólisis (Lifton, 2000). En el extendido hemático se encuentra un hematocrito menor al 20\% (Reimer et al., 2000), además la anemia es macrocitíca hipocrómica muy regenerativa (Lopez, 2004) con policromasia y 
esferocitosis (Moreno, 2003), también se evidencia leucocitosis por neutrofilia con bandemia y monocitosis (Nelson y Couto, 2000).

En el plasma se puede observar hemoglobinemia o bilirrubinemia. La autoaglutinación es prominente en algunos pacientes y se encuentra trombocitopenia en aquellos con síndrome de Evans o coagulación intravascular diseminada (CID). "La presencia de policromasia con autoaglutinación y esferocitosis en un perro con enfermedad y anemia de comienzo agudo es indicativo de AHIM y considerado como diagnóstico; en tal caso no suele necesitarse la prueba de Coombs directa" (Lifton, 2000).

También se puede realizar la prueba de aglutinación directa; para esta prueba se colocan 2 gotas de sangre anticoagulada con EDTA sobre una lamina portaobjetos y se mezcla una de las mismas con 1 gota de solución salina como control y se observa en busca de aglutinación (Cowell et al. 2003). La aglutinación positiva es altamente sugestiva de AHIM.

Reacción de Coombs directa. Esta prueba detecta los anticuerpos sobre las membranas eritrocitarias, empleando un anticuerpo dirigido contra la $\lg G$, $\lg \mathrm{M}$ y complemento (C3b) caninos; en perros con AHIM el reactivo de Coombs (Ig canina) se une a las moléculas de $\lg G$, IgM o complemento, ya ligadas a la superficie eritrocitaria, formando puentes y causando aglutinación, dando la prueba de Coombs como positiva (Mills, 1997).

Pruebas complementarias, bioquímica sérica. Las anormalidades más comunes en la bioquímica son el reflejo del daño producido por la hemólisis, la deshidratación y la hipoxia tisular (Miller, 2000). En la Tabla 2 se citan los hallazgos de laboratorio más frecuentes.

\section{COMPLICACIONES}

Las complicaciones primarias de la AHIM son la coagulación intravascular diseminada (CID) y la enfermedad tromboembólica, en particular el tromboembolismo pulmonar (TEP) (Lifton, 2000). La coagulación intravascular 
diseminada (CID), se refiere a un complejo síndrome en el cual una excesiva coagulación intravascular lleva a una microtrombosis multiorgánica y sangrado paradójico causado por la inactivación o excesivo consumo de plaquetas y factores de la coagulación secundariamente a una fribrinólisis (Couto, 2004). Asimismo, el tromboembolismo pulmonar (TEP) suele ser la complicación más común de la AHIM, esta puede originarse a partir de una coagulación intravascular diseminada (CID) (Álvarez, 2006). Los trombos suelen alojarse en el pulmón, pero se pueden encontrar en múltiples órganos (riñón, hígado, etc) (Lifton, 2000).

Tabla 2. Hallazgos de laboratorio en 17 perros con AHIM

\begin{tabular}{ccc}
\hline Parámetro & Promedio $\pm(\mathrm{SD})$ & Rango \\
\hline Hematocrito $(\%)$ & $15,7 \pm(6,3)$ & $6-35$ \\
VCM $(\mathrm{fl})$ & $78,4 \pm(11,9)$ & $60-129$ \\
Rto Reticulocitos $\left(\times 10^{3} / \mathrm{mcl}\right)$ & $173,7 \pm(188,6)$ & $0,1-102,5$ \\
Leucocitos $\left(\times 10^{3} / \mathrm{mcl}\right)$ & $31,0 \pm(21,1)$ & $5,4-109,5$ \\
Rto Plaquetario & $185 \pm(170)$ & $1-922$ \\
Bilirrubina $(\mathrm{mg} / \mathrm{dl})$ & $7,2 \pm(13,2)$ & $0,01-636$ \\
BUN $(\mathrm{mg} / \mathrm{dl})$ & $31,5 \pm(23,4)$ & $8-85$ \\
Creatinina $(\mathrm{mg} / \mathrm{dl})$ & $0,78 \pm(0.36)$ & $0,3-1,6$ \\
Glucosa $(\mathrm{mg} / \mathrm{dl})$ & $94,5 \pm(26,2)$ & $37-128$ \\
Calcio $(\mathrm{mg} / \mathrm{dl})$ & $9,0 \pm(0,6)$ & $8,1-10,4$ \\
Fósforo $(\mathrm{mg} / \mathrm{dl})$ & $5,0 \pm(1,6)$ & $3,2-9$ \\
ALP $(\mathrm{Ul} / \mathrm{L})$ & $792,2 \pm(1344,2)$ & $21-5570$ \\
ALT $(\mathrm{Ul} / \mathrm{L})$ & $132,2 \pm(251,8)$ & $20-1072$ \\
Albúmina $(\mathrm{g} / \mathrm{dl})$ & $3,03 \pm(0,48)$ & $2,3-3,8$ \\
Globulina $(\mathrm{g} / \mathrm{dl})$ & $2,99 \pm(0,86)$ & $2,1-6$ \\
TCO $2(\mathrm{mEq} / \mathrm{L})$ & $14,7 \pm(4,1)$ & $7,2-21,8$
\end{tabular}

SD: Desviación estándar

VCM: Volumen corpuscular medio

ALP: Fosfatasa alcalina

Fuente: Miller, (2000)
ALT: Alanino aminotransferasa

BUN: Nitrógeno ureico sanguíneo

$\mathrm{TCO}_{2}$ : Dióxido de carbono 


\section{TRATAMIENTO MÉDICO}

Además de controlar o eliminar la enfermedad primaria el tratamiento de la AHIM canina se basa en la administración de corticosteroides con o sin alquilantes, junto a la terapia de sostén necesaria en cada uno de los casos. En la Tabla 3 se resumen los principales medicamentos para el tratamiento de la AHIM canina.

Tabla 3. Principales medicamentos para el tratamiento de la AHIM canina.

\begin{tabular}{|c|c|c|}
\hline Medicamento & $\begin{array}{l}\text { Dosis, Frecuencia y Vía } \\
\text { Administración }\end{array}$ & Observación \\
\hline Prednisolona & 0,2-4 mg/kg/12-24 h, V.O. & $\begin{array}{l}\text { Tratamiento de elección, realizar } \\
\text { monitoreo de Hematocrito cada } 3 \text { días y } \\
\text { ajustar dosis }\end{array}$ \\
\hline Dexametasona & 0,1-0,6 mg/kg I.V. Única dosis & No utilizar en tratamientos prolongados \\
\hline Azatioprina & $\begin{array}{l}50-75 \mathrm{mg} / \mathrm{m}^{2} / 24 \mathrm{~h} \text { V.O. por } 1 \\
\text { semana, luego cada } 48 \text { o } 2 \\
\mathrm{mg} / \mathrm{kg} / \mathrm{día} \text { V.O. con reducción } \\
\text { hasta } 0,5-1 \mathrm{mg} / \mathrm{kg} / 48 \mathrm{~h} .\end{array}$ & $\begin{array}{l}\text { Acción retardada ( } 2-4 \text { semanas post } \\
\text { administración) }\end{array}$ \\
\hline Ciclofosfamida & $\begin{array}{c}50 \mathrm{mg} / \mathrm{m}^{2} / \text { día V.O. por } 4 \text { días } \\
\text { a la semana o } 200-300 \mathrm{mg} / \mathrm{m}^{2} \\
\text { I.V. o V.O. una vez por } \\
\text { semana }\end{array}$ & $\begin{array}{l}\text { Tratamiento eficaz en la AHIM canina, } \\
\text { actúa de } 1 \text { a } 3 \text { semanas post } \\
\text { administración }\end{array}$ \\
\hline Danazol & 5-10 mg/kg/12 h V.O. & $\begin{array}{l}\text { Actúa de } 1 \text { a } 2 \text { semanas post } \\
\text { administración }\end{array}$ \\
\hline Ciclosporina A & 7-15 mg/kg/día V.O o I.V. & $\begin{array}{l}\text { Uso en perros con AHIM refractaria } \\
\text { Vía intravenosa puede generar } \\
\text { anafilaxia }\end{array}$ \\
\hline
\end{tabular}

\section{TRATAMIENTO QUIRÚRGICO (ESPLENECTOMÍA)}

La esplenectomía debe realizarse con cautela ya que el bazo aumentado de tamaño es una fuente importante de hematopoyesis extramedular. La esplenectomía no solo disminuye la eliminación extravascular de eritrocitos, sino que también elimina una importante fuente de producción de anticuerpos (Thompson, 1996). 


\section{MONITOREO DEL PACIENTE}

Al estabilizar el hematocrito por encima del $25 \%$ el paciente puede recibir el alta hospitalaria, este debe ser monitoreado por el propietario, es importante medir el hematocrito, monitorear el hemograma y los reticulocitos antes de reducir la posología de la medicación. Si el paciente muestra efectos colaterales marcados por la corticoterapia la medicación puede ser reducida un poco después de 2 semanas, o se añade otro medicamento (Azatioprina o Danazol). Una vez que la enfermedad entra en remisión la dosis de la prednisolona se puede reducir un 25 a $35 \%$ cada 3 o 4 semanas hasta alcanzar la dosis mínima que mantenga la condición bajo control (Lifton, 2000).

\section{PRONÓSTICO}

La mortalidad global de los pacientes caninos con AHIM varía del 20 al $50 \%$ pero puede ser más elevada hasta $80 \%$ (Reimer et al., 2000), como es el caso de perros con complicaciones y enfermedades subyacentes que son de pronóstico reservado.

\section{CONCLUSIONES}

La anemia hemolítica inmunomediada canina es un padecimiento que puede variar de signo clínico de una enfermedad "inicial" a una propiamente dicha, por lo tanto, su presentación clínica es muy variada y en ocasiones se pueden evidenciar signos que distraen al médico veterinario del diagnóstico.

El único hallazgo microscópico que indica que el padecimiento de la anemia hemolítica es de tipo inmunomediado, es la presencia de esferocitosis y aglutinación en el extendido hemático, o la comprobación de la existencia de anticuerpos sobre las membranas eritrocitarias caninas por medio de la reacción de Coombs directa.

Se considera como diagnostico la AHIM la presencia de policromasia con autoaglutinación y esferocitosis en un perro con enfermedad y anemia de 
comienzo agudo. Se ha reportado con mayor frecuencia en hembras y en algunas razas en específico, lo que denota una susceptibilidad de tipo genético y la influencia hormonal sobre el sistema inmune.

Existen medicamentos efectivos para el control y tratamiento de la AHIM en perros; sin embargo, deben ser suministrados con precaución debido a sus graves efectos colaterales.

\section{REFERENCIAS BIBLIOGRÁFICAS}

1. Álvarez F. Seminario de Medicina Interna Oncológica. Diagnostico y manejo del paciente con anemia y trombocitopenia inmunomediada. Bogotá: AgostoSeptiembre, p. 12-16. 2006.

2. Barker R. N., Shen C. R., Elson C. J. T cell specificity in murine haemolytic anemia induced by rat red blood cells. Clin Exp Immunol., 129 (2): 208-213. 2002.

3. Buch B. Interpretación de los Análisis de Laboratorio para Clínicos de Pequeños Animales. Madrid, España: Ediciones Harcourt S.A., p 39-91. 1999.

4. Cerecedo M. A.; Rivera L.; Lara O. Anemia hemolítica inmunomediada en un perro. Memorias Cuarta Jornada De Patología Clínica Veterinaria UNAM. México, p 1-4. 2004.

5. Couto C. G. Diagnóstico y Tratamiento de Anemias I y II, Seminario de Oncología, VEPA. Cali. p 2-3. Octubre 1997.

6. Couto C. G. Coagulación intravascular diseminada en perros y gatos. Memorias IX Congreso de Médicos Veterinarios en Pequeñas Especies del Bajio. Ciudad de México, p 1-15. 2004.

7. Corato A., Shen C. R., Mazza G., Barker R. N., Day M. J. Proliferative responses of peripheral blood mononuclear cells from normal dogs and dogs with autoimmune haemolytic anemia to red blood cell antigens. Vet Immunol Immunopathol., 59 (3-4): 191-204. 1997.

8. Cowell R. L.; Tyler R. D.; Meinkoth J. H. Citología y hematología diagnóstica en el perro y el gato. Segunda edición. Barcelona, España: Multimédica, $p$ 263-301. 2003.

9. Day M. J. Atlas en color de enfermedades inmunomediadas del perro y el gato: Cuadros clínicos, diagnóstico y tratamiento. Madrid, España: Ediciones Grass, p 9-87. 1999.

10. Day M. J.; Mackin A. Manual of Canine and Feline Haematology and Transfusion. En: Brit Small and Vet Assoc. p 135-155. 2000.

11. Herrera M.; Estrada M. Esferocitosis hereditaria: aspectos clínicos, Bioquímicos y Moleculares. Revista Cubana de Hematología, Inmunología y Hemoterapia, 18 (1): 7-24. 2003.

12. Illanes J., Ríos A. Anemia hemolítica en un perro. Caso clínico, Hospitales Veterinarios, 5 (3): 71-79. 2013. 
13. Lifton J. S. Manejo de la anemia hemolítica inmunomediada canina. Selecciones Veterinarias. 8 (1): 81-84. 2000.

14. López C. A. Insuficiencia renal aguda, colangiohepatitis, anemia hemolítica inmunomediada y pancreatitis en un perro. Memorias Cuarta Jornada de Patología Clínica Veterinaria UNAM. México. p 1-5. 2004.

15. Manz R. A., Moser K., Burmester G. R., Radbruch A., Hiepe F. Immunological memory stabilizing autoreactivity. Curr Top Microbiol Immunol., (305): 241-257. 2006.

16. Miller E. CVT Update: Diagnosis and Treatment of immune-mediated hemolytic anemia. Kirk's Current Veterinary Therapy XIII, J Small Anim Pract. Collins, Colorado: WB Saunders Company, p 427-434. 2000.

17. Mills J. M. Compensated immune mediated haemolytic anaemia in a dog. Aust Vet J., 75 (1): 24-26. 1997.

18. Moreno C. F. Determinación de parámetros hematológicos normales en una población de caninos y felinos de una clínica veterinaria al norte de la ciudad de Bogotá. Trabajo de Grado Bacteriología, Facultad de Ciencias, Pontificia Universidad Javeriana. Bogotá. p 32. 2003.

19. Nassiri M. S.; Shirani D.; Khazrainia K.; Hajmohammadali A.; Sharifi H.; The investigation of the prevalence of immune-mediated hemolytic anemia (IMHA) in anemic dogs referred to the Veterinary Teaching Hospital of the University of Tehran. Comparative Clinical Pathology, 14 (3): 121-124. 2005.

20. Nelson W. R. and Couto C.G. Medicina Interna de Pequeños Animales; Buenos Aires, República de Argentina: Intermedica, p 1244-1247, 1304, 13101312. 2000.

21. Olarte W. Seminario Nacional, Hematología en pequeños animales. Anemia Hemolítica Inmunomediada. Bogotá, p 2-35. Julio 2004.

22. Paz A. Anemia hemolítica inmunomediada en perros. Memorias Cuarta Jornada de Patología Clínica Veterinaria UNAM. México, p 1-9. 2004.

23. Rebar A. H. Interpretación del hemograma canino y felino. Eritrocitos en periodo de salud y enfermedad. Wilmington, Delaware: The Gloyd Group. Inc., p 21-30. 2003.

24. Reimer M., Troy G., Warnick L. Anemia hemolítica inmunomediada, Selecciones Veterinarias, 8 (5): 524-526. 2000.

25. Semple J. W.; Freedman J. Autoimmune pathogenesis and autoimmune hemolytic anemia. Semin Hematol., 42 (3): 122-130. 2005.

26. Thompson J. P. Manual Clínico de Pequeñas Especies Birchard S. J., Sherding R. G. Enfermedades Sistémicas Inmunomediadas, Anemia Hemolítica Inmunomediada. Vol. 1. Interamericana McGraw-Hill, p 204-207. 1996.

27. Tizard I. R. Inmunología veterinaria. Sexta edición. México: McGraw-Hill Interamericana, p. 10-18, 22-26, 50-114, 150-160, 174-194, 351-359, 392-399, 411-413. 2002.

28. Wilkerson M. J., Davis E., Shuman W., Harkin K., Cox J., Rush B. Isotypespecific antibodies in horses and dogs with immune-mediated hemolytic anemia. J Vet Med., 14 (2): 190-196. 2000. 